\section{New Antilipid Drugs: Do the Known Benefits Justify the Expense?}

\section{THE "PRO" SIDE}

A new drug that costs $\$ 300$ per dose is expensive no matter which way you look at it. For a lipid-lowering drug, therapy will continue for the long term, possibly 20 or more years, so the cost would be $\$ 150000$ or more for a single patient. An initial response might be that such a drug is too expensive to be covered by a government drug program. However, we need to look beyond cost alone and consider the possible benefits.

The proprotein convertase subtilisin/kexin type 9 (PCSK9) inhibitors are a new class of lipid-lowering medications. Evolocumab (Repatha) received its notice of compliance from Health Canada in September 2015, and alirocumab (Praluent) received its notice in April 2016. ${ }^{1}$ The bench-to-bedside journey of this class of medications was rapid. PCSK9 was identified and characterized in 2003, and it was only in 2006 that it was implicated in the regulation of cholesterol. ${ }^{2}$ Now, just 10 years later, we have 2 new medications for lowering lowdensity-lipoprotein cholesterol (LDL-C).

To date, the efficacy data (i.e., the impact on clinical outcomes) come from surrogate marker trials, which have measured the effects of PCSK9 inhibitors on the lipid profile, particularly LDL-C. Each of the 2 above-mentioned agents is able to provide an additional 50\%-60\% lowering of LDL-C, on top of existing therapy such as statins. Although the primary objective of these trials was to determine the effect on the lipid profile, these studies also provided an opportunity to explore the impact of these agents on cardiovascular outcomes. The OSLER 1 and 2 trials included patients who were receiving evolocumab or placebo over a median of 11.1 months. ${ }^{3}$ At baseline, $20 \%$ of the patients had established coronary artery disease and the remainder had cerebrovascular or peripheral vascular disease or were at high risk of cardiovascular disease. At 1 year, the rate of cardiovascular events was $0.95 \%$ in the group that had been randomly assigned to receive evolocumab and $2.18 \%$ among those assigned to received placebo (hazard ratio $0.47,95 \%$ confidence interval [CI] $0.28-0.78 ; p=0.003)$. Similarly, in the ODYSSEY trial, the outcomes of patients taking alirocumab were better than those who were taking placebo over a 78 -week period ${ }^{4}$; the rate of major cardiovascular events was $1.7 \%$ among those who received alirocumab and 3.3\% among those receiving placebo (hazard ratio 0.52 , 95\% CI $0.31-0.90 ; p=0.02$ ). Thus, these 2 trials, albeit limited by relatively small sample sizes and short-term follow-up, showed that both of the PCSK9 inhibitors had a significant impact on important outcomes. Larger, longer-term trials are now in progress, with results expected in 2017 and 2018. 5,6 I believe that the results from these trials will reflect those seen in the smaller, surrogate marker trials and that PCSK9 inhibitors will be associated with significant reductions in important cardiovascular outcomes, such as death due to cardiovascular disease, myocardial infarction, and stroke. It is from this assumption that I argue they are worth their cost.

It is important to consider the patients who would be denied this potentially life-saving medication if the decision were to be made on the basis of cost alone. One patient group to consider is patients with familial hypercholesterolemia, with LDL-C above $5 \mathrm{mmol} / \mathrm{L}$ and physical findings such as tendon xanthoma. These patients are at a 20-fold greater risk of cardiovascular disease if untreated. ${ }^{7}$ Even when treated with currently available therapies, these individuals are unable to reach target LDL-C levels. The new class of medications offers them an opportunity to achieve guideline-recommended targets and presumably better quality of life and longer life expectancy. Another group of patients currently at risk of cardiovascular-related morbidity and mortality are those who cannot take statins. Statins are effective in reducing mortality among patients with cardiovascular disease and are generally safe. However, despite the drugs being well tolerated by most patients, some individuals experience adverse effects. Some of these adverse effects can be bothersome (e.g., insomnia), whereas others are associated with significant harm (e.g., autoimmune myopathy). ${ }^{8,9}$ Patients in both of these situations are unable to derive the benefits of statins, and there are currently no alternative medications to reduce their risk of events such as heart attacks; as such, lifestyle changes are the only option.

The scenario of trying to use expensive therapies in an environment with limited budgets is not new in the world of Canadian pharmacy. Alteplase and tenecteplase ( $\$ 2700$ per dose) are routinely used to treat myocardial infarction and strokes. A course of rituximab for rheumatoid arthritis, pertuzumab for breast cancer, or ledipasvir-sofosbuvir for hepatitis $\mathrm{C}$ can cost tens of thousands of dollars, and these drugs are funded. What has allowed these expensive medications to be used? They have been rationed. Individuals who are thought to derive the greatest 
benefit (e.g., those who have had no response to or are unable to take other therapies) are identified, and the cost of the medications is covered. A similar approach will be needed for the PCSK9 inhibitors, such that they are rationed to those who will benefit the most. This approach was recommended by the Institute for Clinical and Economic Review, a US-based group that provides independent review of effectiveness and value. ${ }^{10}$ Of interest, such rationing has started to take place as lipid guidelines are updated. The recent American College of Cardiology expert consensus statement suggests that PCSK9 inhibitors should be considered in these 2 populations. ${ }^{11}$

Although funding decisions are typically made at a distance from patient care, front-line pharmacists play an important role in optimizing the care of patients with lipid disorders. In their day-to-day care of such patients, pharmacists should ensure that statins are being used optimally, by determining whether patients are receiving the correct dose when a statin is indicated or identifying patients who have been deemed statin intolerant. The latter situation should prompt the pharmacist to conduct an assessment to identify possible causes, particularly reversible ones, of statin intolerance, to ensure that the patient is truly statin intolerant. ${ }^{8,11}$ Pharmacists also play an important role in stewardship of the drug budget, looking for irrational or out-of-date prescribing. The Choosing Wisely Canada campaign (www.choosingwisely canada.org/) recognizes irrational prescribing. Although many of the Choosing Wisely recommendations focus on unnecessary tests and procedures, some are medication related. This campaign provides an opportunity and framework to engage our physician and other prescriber colleagues in projects to optimize prescribing. In turn, this may allow funds saved by reducing inappropriate prescribing to be reallocated to pay for PCSK9 inhibitors.

The PCSK9 inhibitors are very expensive, and some may say they are not worth the money. Although it may be hard to justify their use on a widespread basis, I believe there are patients for whom the benefit of these medications will justify the cost.

\section{References}

1. Health Canada drug product database. Ottawa (ON): Health Canada; 2015 Jul [cited 2016 May 22]. Available from: http://webprod5.hc-sc.gc.ca/ dpd-bdpp/index-eng.jsp

2. Bergeron N, Phan BAP, Ding Y, Fong A, Krauss RM. Proprotein convertase subtilisin/kexin type 9 inhibition: a new therapeutic mechanism for reducing cardiovascular disease risk. Circulation. 2015;132(17):1648-66.

3. Sabatine MS, Giugliano RP, Wiviott SD, Raal FJ, Blom DJ, Robinson J, et al; Open-Label Study of Long-Term Evaluation against LDL Cholesterol (OSLER) Investigators. Efficacy and safety of evolocumab in reducing lipids and cardiovascular events. N Engl J Med. 2015;372(16):1500-9.

4. Robinson JG, Farnier M, Kremph M, Bergeron J, Luc G, Averna M, et al.; ODYSSEY LONG TERM Investigators. Efficacy and safety of alirocumab in reducing lipids and cardiovascular events. N Engl J Med. 2015; 372(16):1489-99.

5. Schwartz GG, Bessac L, Berdan LG, Bhatt DL, Bittner V, Diaz R, et al. Effect of alirocumab, a monoclonal antibody to PCSK9, on long-term cardiovascular outcomes following acute coronary syndromes: rationale and design of the ODYSSEY outcomes trial. Am Heart J. 2014;168(5):682-9.

6. Sabatine MS, Giugliano RP, Keech A, Honarpour N, Wang H, Liu T, et al. Rationale and design of the Further cardiovascular OUtcomes Research with PCSK9 Inhibition in subjects with Elevated Risk trial. Am Heart J. 2016;173:94-101.
7. Genest J, Hegele RA, Bergeron J, Brophy J, Carpentier A, Couture P, et al. Canadian Cardiovascular Society position statement on familial hypercholesterolemia. Can J Cardiol. 2014;30(12):1471-81.

8. Mancini GBJ, Baker S, Bergeron J, Fitchett D, Frohlich J, Genest J, et al. Diagnosis, prevention, and management of statin adverse effects and intolerance: proceedings of a Canadian working group consensus conference. Can J Cardiol. 2011;27(5):635-62.

9. Mammen AL. Statin-associated autoimmune myopathy. $N$ Engl J Med. 2016;374(7):6649.

10. Tice JA, Ollendorf DA, Cunningham C, Pearson SD, Kazi DS, Coxson PG, et al. PCSK9 inhibitors for treatment of high cholesterol: effectiveness, value, and value-based price benchmarks. Final report. Boston (MA): Institute for Clinical and Economic Review; 2015 Nov 24 [cited 2016 May 22]. Available from: http://icer-review.org/material/high-cholesterol-final-report/

11. Lloyd-Jones DM, Morris PB, Ballantyne CM, Birtcher KK, Daly DD Jr, DePalma SM, et al. 2016 ACC expert consensus decision pathway on the role of non-statin therapies for LDL-cholesterol lowering in the management of atherosclerotic cardiovascular disease risk: a report of the American College of Cardiology Task Force on Clinical Expert Consensus Documents. J Am Coll Cardiol. 2016;68(1):92-125.

Heather Kertland, BScPhm, PharmD, FCSHP

Clinical Pharmacy Specialist/Leader, St Michael's Hospital

Assistant Professor (Status only), University of Toronto

Toronto, Ontario

Competing interests: None declared

\section{THE "CON" SIDE}

It is well established that low-density lipoproteins play a causal role in the development of atherosclerosis and that reduction of low-density lipoprotein cholesterol (LDL-C) with a statin is a cornerstone in the prevention of cardiovascular disease. ${ }^{1}$ However, there remains an unmet need to reduce the residual risk for patients who do not reach target LDL-C levels on maximally tolerated statin alone or in combination with ezetimibe. Consequently, the recent Health Canada approval of 2 cholesterol-lowering drugs from a new class, the monoclonal antibody proprotein convertase subtilisin/kexin 9 (PCSK9) inhibitors, nearly 3 decades after approval of the first statin, is exciting. These agents, evolocumab (Notice of Compliance September 9, 2015) and alirocumab (Notice of Compliance April 11, 2016), are indicated as "an adjunct to diet and maximally tolerated statin therapy for the treatment of adults with heterozygous familial hypercholesterolemia $(\mathrm{HeFH})$ or clinical atherosclerotic cardiovascular disease, who require additional lowering of LDL-C".,3

In phase III randomized clinical trials, both of these PCSK9 inhibitors have been associated with reductions in LDL-C of between $50 \%$ and $70 \%$ when added to statin therapy. 4,5 Although lowering LDL-C was the primary outcome of all PCSK9 inhibitor trials published to date, cardiovascular event data were collected as a secondary outcome, and a meta-analysis has shown a $50 \%$ reduction in the odds of death from cardiovascular disease (odds ratio $[\mathrm{OR}] 0.50,95 \%$ confidence interval $[\mathrm{CI}] 0.23-1.10$ ) and 
myocardial infarction (OR 0.49, 95\% CI 0.26-0.93). ${ }^{6}$ However, there was also a nonsignificant doubling in the odds of stroke (OR 1.97, 95\% CI 0.69-5.65). ${ }^{6}$ These results must be interpreted cautiously because they reflect combined results from studies that evaluated different drugs, different dosing schedules, different patient populations, and different comparison groups (placebo and ezetimibe).

Beyond the surrogate end point of a large reduction in lipids and speculation about improved cardiovascular outcomes for patients, practitioners must consider the cost-effectiveness of these novel agents. A recent report issued by the Institute for Clinical and Economic Review provides the potential budget impact, on the US health care system, of using PCSK9 inhibitor monoclonal antibodies. ${ }^{7}$ Based on available data, estimates of LDL-C level lowering were the key inputs for this cost-effectiveness analysis, which used a validated model of coronary heart disease and stroke in the US population over 35 years of age. The investigators estimated the benefits and costs for individuals in 3 specific highrisk populations. The benefits were reported as the number needed to treat for 5 years with a PCSK9 inhibitor to prevent 1 major adverse cardiac event, with cost estimates reported as the incremental cost-effectiveness ratio at current US prices (about US\$14 000 annually) per quality-adjusted life-year gained. The estimates derived from this model are summarized in Table 1. Additionally, using estimates for the magnitude of improvement in patient outcomes and a threshold cost required to avoid excessive cost burdens to the US health care system, another report from the same analysis ${ }^{8}$ suggested that the annual price of the PCSK9 inhibitors would need to be US $\$ 2177$ to enable use without limitation by eligible patients. As a result, the investigators concluded that PCSK9 inhibitors had low value, because of their poor long-term cost-effectiveness and short-term affordability.

Although similar economic modelling has not been done in Canada, it is safe to assume from this US analysis that achieving cost-effectiveness at an acceptable threshold would require a significant price reduction from the current cost of these drugs in Canada (about $\$ 7200 /$ year). More importantly, the estimated benefits of these drugs warrant skepticism, because they are based upon LDL-C lowering as a surrogate for cardiovascular risk and the evaluation of secondary end points with very low event rates in clinical trials. Although LDL-C lowering is widely accepted as a surrogate marker for cardiovascular risk in statin-treated patients, on the basis of countless large statin trials, this assumption may not apply to the PCSK9 inhibitors because the use of surrogates must be validated for each drug class. ${ }^{9}$ Without knowing the true clinical benefits of these drugs from the large outcome trials that are in progress (with results expected in the next 1-2 years), as well as our current lack of insight regarding their long-term safety, the prudent practitioner must conclude that the benefits of the PCSK9 inhibitors do not yet justify their expense in all potentially eligible patient populations.
Table 1. Effectiveness and Value of PCSK9 Inhibitors ${ }^{7,8}$

\begin{tabular}{lcc} 
High-Risk Population & $\begin{array}{c}\mathrm{NNT}_{5} \text { to Prevent } \\
\text { 1 Major Adverse } \\
\text { Cardiac Event }\end{array}$ & $\begin{array}{c}\text { ICER/QALY } \\
\text { Gained (\$US) }\end{array}$ \\
\hline Patients with HeFH & 28 & 290000 \\
\hline $\begin{array}{l}\text { Patients with CVD } \\
+ \text { statin intolerance }\end{array}$ & 21 & 274000 \\
\hline $\begin{array}{l}\text { Patients with CVD } \\
+ \text { LDL-C } \geq 1.8 \text { mmol/L }\end{array}$ & 302000 \\
on statin & 21 \\
\hline CVD = cardiovascular disease, HeFH = heterozygous familial \\
hypercholesterolemia, ICER = incremental cost-effectiveness \\
ratio, LDL-C = low-density-lipoprotein cholesterol, \\
NNT $=$ number needed to treat for 5 years, \\
PCSK9 = proprotein convertase subtilisin/kexin 9, \\
QALY = quality-adjusted life-year.
\end{tabular}

The more pragmatic approach would be to limit the utilization of these agents to the highest-risk patients and those most likely to derive the greatest benefit from their use. Given that patients with $\mathrm{HeFH}$ are characterized by significantly elevated levels of LDL-C ( $>4.9 \mathrm{mmol} / \mathrm{L})$ and a high risk of premature coronary artery disease, this population may be considered appropriate for treatment with PCSK9 inhibitors, on the basis of published data. This approach is supported by the recommendations of the Common Drug Review (CDR) for evolocumab (the alirocumab report is in progress). The main CDR recommendation was to list evolocumab as an adjunct to diet, exercise, and maximally tolerated statin therapy (with or without ezetimibe) among adults with $\mathrm{HeFH}$ who require additional therapy to achieve the LDL-C target of less than $2.0 \mathrm{mmol} / \mathrm{L} .{ }^{10}$ On the basis of pharmacoeconomic modelling conducted as part of the CDR process, the expert committee concluded that evolocumab was a cost-effective treatment option for patients with HeFH who are unable to achieve target LDL-C. ${ }^{10}$ The CDR also recommended that evolocumab not be listed as adjunctive therapy for adult patients with clinical atherosclerotic cardiovascular disease who require additional LDL-C lowering, noting that this drug was not considered to be cost-effective in this population. ${ }^{10}$

The real answer to the question of whether the known benefits associated with the PCSK9 inhibitors justify their expense is a moving target. From currently available data, it appears that these agents are not cost-effective, except in a specific subset of at-risk patients-people with $\mathrm{HeFH}$ who are already receiving maximally tolerated statin therapy, with or without ezetimibe, and who have not achieved an LDL-C target of less than $2.0 \mathrm{mmol} / \mathrm{L}$. Nevertheless, data from the large, ongoing outcome trials with the PCSK9 inhibitors, as well as information regarding their long-term safety, will further inform this important question in the next few years.

\section{References}

1. Anderson TJ, Grégoire J, Hegele RA, Couture P, Mancini GB, McPherson R, et al. 2012 update of the Canadian Cardiovascular Society guidelines for the diagnosis and treatment of dyslipidemia for the prevention of cardiovascular disease in the adult. Can J Cardiol. 2013;29(2):151-67. 
2. Repatha ${ }^{\mathrm{TM}}$ (evolocumab) [product monograph]. Mississauga (ON): Amgen Canada Inc; 2015 Sep 10.

3. Praluent ${ }^{\mathrm{TM}}$ (alirocumab) [product monograph]. Laval (QC): Sanofi-aventis Canada Inc; 2016 Apr 11.

4. Sabatine MS, Giugliano RP, Wiviott SD, Raal FJ, Blom DJ, Robinson J, et al.; Open-Label Study of Long-Term Evaluation against LDL Cholesterol (OSLER) Investigators. Efficacy and safety of evolocumab in reducing lipids and cardiovascular events. N Engl J Med. 2015;372(16):1500-9.

5. Robinson JG, Farnier M, Krempf M, Bergeron J, Luc G, Averna M, et al.; ODYSSEY LONG TERM Investigators. Efficacy and safety of alirocumab in reducing lipids and cardiovascular events. $N$ Engl J Med. 2015; 372(16):1489-99.

6. Navarese EP, Kolodziejczak M, Schulze V, Gurbel PA, Tantry U, Lin Y, et al. Effects of proprotein convertase subtilisin/kexin type 9 antibodies in adults with hypercholesterolemia: a systematic review and meta-analysis. Ann Intern Med. 2015;163(1):40-51.

7. Tice JA, Ollendorf DA, Cunningham C, Pearson SD, Kazi DS, Coxson PG, et al. PCSK9 inhibitors for treatment of high cholesterol: effectiveness, value, and value-based price benchmarks: final report. Boston (MA): Institute for Clinical and Economic Review; 2015 Nov 24 [cited 2016 Jul 8]. Available from: http://icer-review.org/wp-content/uploads/2015/04/ FinalReport-for-Posting-11-24-15.pdf

8. Tice JA, Kazi DS, Pearson SD. Proprotein convertase subtilisin/kexin type 9 (PCSK9) inhibitors for treatment of high cholesterol levels: effectiveness and value. JAMA Intern Med. 2016;176(1):107-8.
9. Rodriguez-Gutierrez R, Shah ND, Montori VM. Predicting the overuse of PCSK-9 inhibitors. JAMA. 2015;314(18):1909-10.

10. Common drug review: CADTH Canadian Drug Expert Review Committee final recommendation: evolocumab. Ottawa (ON): Canadian Agency for Drugs and Technology in Health; 2016 Feb [cited 2016 May 24]. Available from: https:/www.cadth.ca/sites/default/files/cdr/complete/SR0441_ complete_Rapatha-Feb-23_16_e.pdf

Glen J Pearson, BSC, BScPhm, PharmD, FCSHP

Division of Cardiology

Mazankowski Alberta Heart Institute

University of Alberta

Edmonton, Alberta

Competing interests: Glen Pearson is the Vice Co-Chair of the committee responsible for the 2016 update of the Canadian Cardiovascular Society guidelines for the diagnosis and treatment of dyslipidemia for the prevention of cardiovascular disease in the adult.

\section{Let's stay connected.}

Renew your membership today!

\section{Restons en contact.}

Renouvelez votre adhésion dès aujourd'hui! advocacy

networking

best practices

education

research

recognition

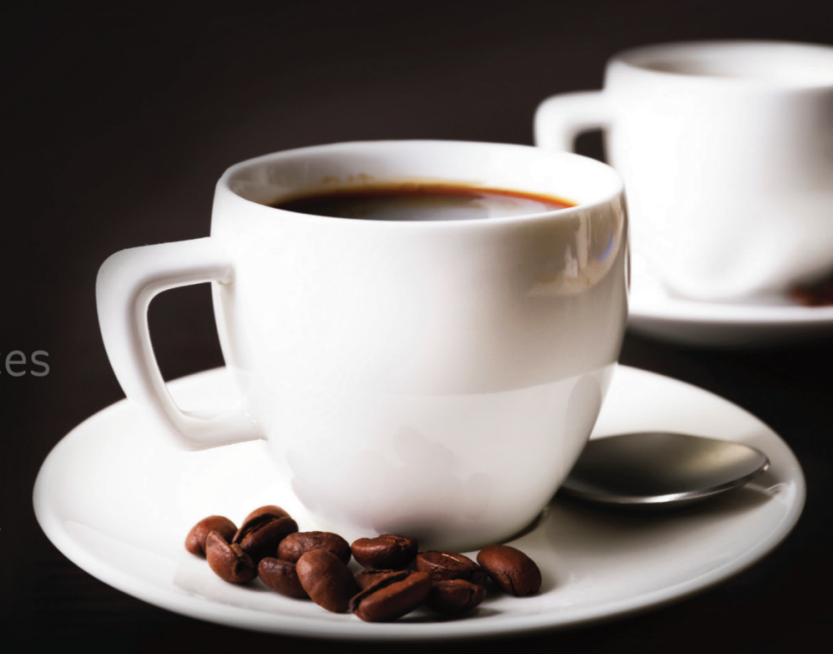

valorisation réseautage meilleures pratiques formation recherche reconnaissance 